\title{
Modifying and using Yates' algorithm
}

\section{Hans Riedwyl}

Received: May 20, 1996; revised version: September 26, 1996

It is well known that Yates' algorithm can be used to estimate the effects in a factorial design. We develop a modification of this algorithm and call it modified Yates' algorithm and its inverse. We show that the intermediate steps in our algorithm have a direct interpretation as estimated level-specific mean values and effects. Also we show how Yates' or our modified algorithm can be used to construct the blocks in a $2^{k}$ factorial design and to generate the layout sheet of a $2^{k-p}$ fractional factorial design and the confounding pattern in such a design. In a final example we put together all these methods by generating and analysing a $2^{6-2}$ design with 2 blocks.

Key words: Yates' algorithm, $2^{k}$ factorial design, fractional factorial design

\section{Introduction}

A $2^{k}$ factorial design is one in which $k$ variables or factors labeled $\mathrm{A}, \mathrm{B}, \mathrm{C}, \mathrm{D}, \ldots$ are each allocated to two levels, conventionally \pm 1 in coded coordinates, and every possible combination of the \pm sign is run, typically in a completely randomized or randomized block order. A fractional two-level design is one that employs only a fraction of the $2^{k}$ runs. Such designs use a $2^{-p}$ fraction of the whole $2^{k}$ runs and therefore have been designated $2^{k-p}$ fractional factorials. The twolevel factorial designs have mainly been used in full and fractional form since Yates (1935). A large compilation of $2^{k-p}$ designs was made available by the National Bureau of Standards (1957). See also Box, Hunter and Hunter (1978), Montgomery (1991) and Daniel (1976). 
Yates' algorithm to estimate the effects and also the reversed form to estimate the residuals in a $2^{k}$ factorial design are well known. We develop a modification of these two algorithms and call them modified Yates' algorithm and its inverse. We show that the intermediate steps in our algorithm have a direct interpretation as estimated levelspecific mean values and effects. Further we show how Yates' or our modified algorithm can be used to construct the blocks in a $2^{k}$ factorial design and to generate the layout sheet of a $2^{k-p}$ fractional factorial design and the confounding pattern in such a design. In a final example we put together all these methods by generating and analysing a $2^{6-2}$ design with 2 blocks. This paper demonstrates the use of an algorithm for the analysis of effects and residuals for full and fractional factorials. The same algorithm can be used for selecting fractions and blocks, and for observing aliasing patterns from factorial layouts.

\section{Classical Yates' Algorithm}

In the following we analyse the results of a $2^{3}$ factorial design, the first replicate of exercise 9-1 by Montgomery (1991) on page 310 . The data are given in Table 1.

Table 1. Data

\begin{tabular}{|c|c|c|c|}
\hline \multicolumn{2}{|c|}{} & $A(-1)$ & $A(+1)$ \\
\hline$C(-1)$ & $B(-1)$ & 22 & 32 \\
\cline { 2 - 4 } & $B(+1)$ & 35 & 55 \\
\hline \multirow{2}{*}{$C(+1)$} & $B(-1)$ & 44 & 40 \\
\cline { 2 - 4 } & $B(+1)$ & 60 & 39 \\
\hline
\end{tabular}

Table 2 shows us the classical Yates' algorithm applied to these data, which are entered in the column "Response" in the standard order (-), A, B, AB, C, AC, BC, ABC. Column (1) consists of the 4 sums and the 4 differences of successive pairs of entries in column "Response". Columns (2) and (3) repeat the same operations on the values in the preceding columns. We get column "Effect" by dividing the first entry of column (3) by 8 and the rest of column (3) by 4 . 
Table 2. Classical Yates' Agorithm

\begin{tabular}{|c|c|c|c|c|c|}
\hline Response & Symbol & $(1)$ & $(2)$ & $(3)$ & Effect \\
\hline 22 & $(-)$ & 54 & 144 & 327 & 40.875 \\
\hline 32 & $\mathrm{~A}$ & 90 & 183 & 5 & 1.25 \\
\hline 35 & $\mathrm{~B}$ & 84 & 30 & 51 & 12.75 \\
\hline 55 & $\mathrm{AB}$ & 99 & -25 & -7 & -1.75 \\
\hline 44 & $\mathrm{C}$ & 10 & 36 & 39 & 9.75 \\
\hline 40 & $\mathrm{AC}$ & 20 & 15 & -55 & -13.75 \\
\hline 60 & $\mathrm{BC}$ & -4 & 10 & -21 & -5.25 \\
\hline 39 & $\mathrm{ABC}$ & -21 & -17 & -27 & -6.75 \\
\hline
\end{tabular}

\section{Modified Yates' Algorithm}

In the left half of Table 3 the same data are analysed with our modified algorithm. The calculations that lead to this table are displayed in the left half of Table 4 in a spreadsheet-like manner. The construction of such tables for different numbers of factors is explained in Appendix A. If we compare Table 2 with the left half of Table 3 we notice that they are closely related: we get columns (1), (2) and (3) of Table 3 by dividing by 2,4 and 8 respectively and reordering the entries of the corresponding columns of Table 2, and of course the columns "Effect" are identical. Yates' algorithm has the advantage that the same calculations can be repeated thrice from one column to the next, whereas the modified algorithm makes different calculations from column to column. But nowadays with the availability of computers, this doesn't make any problems. However, our modified algorithm has a great advantage over Yates': our intermediate columns have an easy interpretation as estimated level-specific mean values and effects, as we will show in the following: From Table 1 it is easy to calculate the estimated mean values and the estimated main effects of $\mathrm{A}$ for each ievel combination of $\mathrm{B}$ and $\mathrm{C}$ :

$\begin{array}{lllll} & \mathrm{B}(-1), \mathrm{C}(-1) & \mathrm{B}(+1), \mathrm{C}(-1) & \mathrm{B}(-1), \mathrm{C}(+1) & \mathrm{B}(+1), \mathrm{C}(+1) \\ \text { mean value } & (22+32) / 2=27 & (35+55) / 2=45 & (44+40) / 2=42 & (60+39) / 2=49.5 \\ \text { half main effect A } & (32-22) / 2=5 & (55-35) / 2=10 & (40-44) / 2=-2 & (39-60) / 2=-10.5\end{array}$

In column (1) of the modified algorithm we find exactly these mean values and half main effects. Similarly the values in column (2) are the estimated mean values, one half of the estimated main effects of $A$ and $B$ and one half of the estimated interaction $A B$ for each level of $\mathrm{C}$ : 

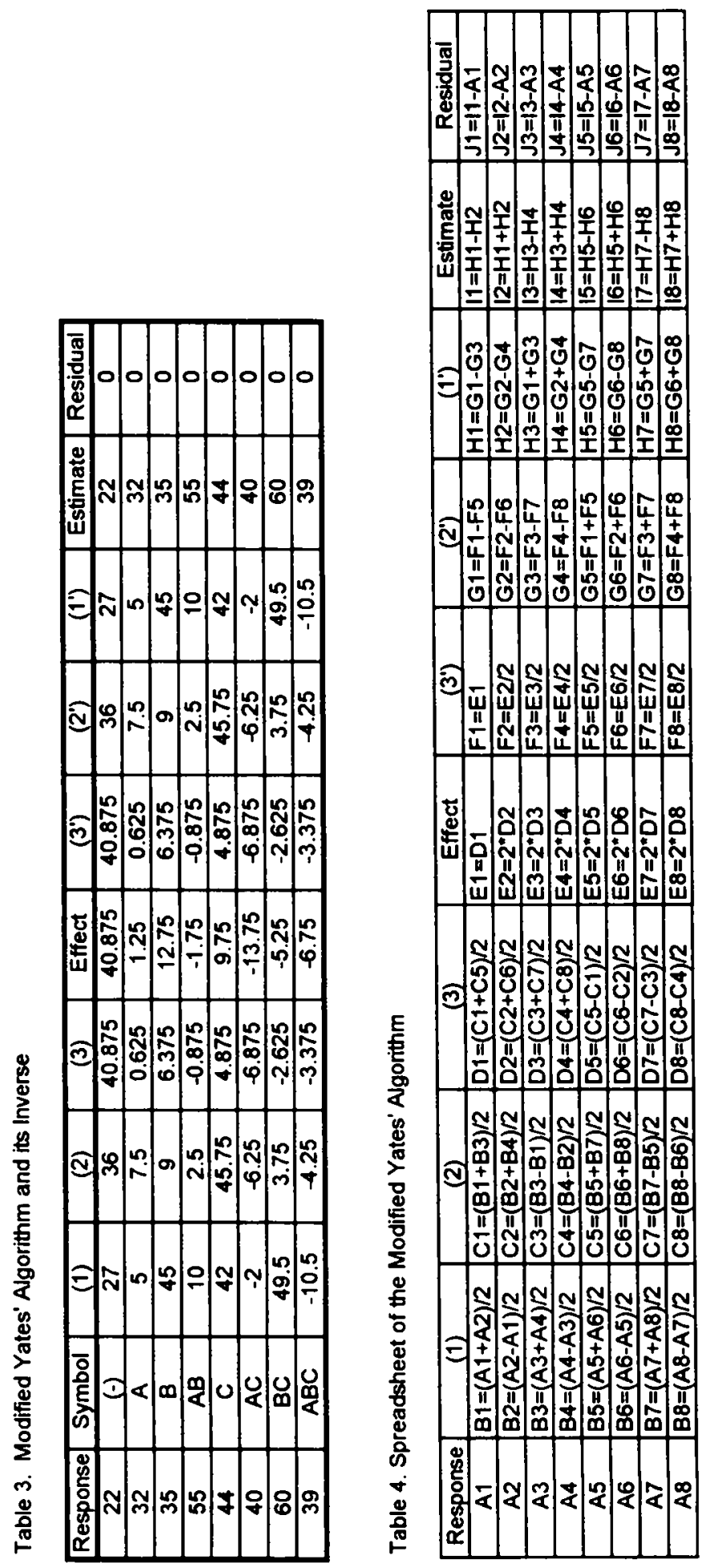


$\begin{array}{lrr} & C(-1) & C(+1) \\ \text { mean value } & (55+35+32+22) / 4=36 & (39+60+40+44) / 4=45.75 \\ \text { half main effect A } & (55-35+32-22) / 4=7.5 & (39-60+40-44) / 4=-6.25 \\ \text { half main effect B } & (55+35-32-22) / 4=9 & (39+60-40-44) / 4=3.75 \\ \text { half interaction AB } & (55-35-32+22) / 4=2.5 & (39-60-40+44) / 4=-4.25\end{array}$

This interpretation that now has been demonstrated for a $2^{3}$ example is also valid for larger designs. We will see this in an example at the end of section 5 .

\section{Inverse Algorithm}

Following a proposal of Daniel (1976) it is possible to reconstruct the original response values from the effect values by writing down the effect values in reverse order and applying Yates' algorithm to this column. With this procedure it is also possible to calculate the residuals that result from setting those effects equal to zero which seem to be insignificant.

We do the same thing with a slight modification of our algorithm. In the right half of Table 3 we see in columns (2') and (1') exactly the same level-specific mean values, half main effects and half interactions as in column (2) and (1). In column "Estimate" we have reconstructed the responses from the effects. In the right half of Table 4 we display the calculations of this inverse algorithm and in Appendix A we explain the similarity between the left and the right side of Table 4, that is between the modified algorithm and its inverse. If we want to eliminate some insignificant effects and calculate the resulting residuals, we just have to write a 0 in the corresponding cell of column (3') and have immediately calculated the residuals as difference between the columns "Response" and "Estimate". (We also could put the 0 in column "Effect", but column (3') has the advantage that we don't erase the estimated effects.)

\section{An Example}

We analyse a single replicate of a $2^{4}$ design, Example $9-2$ by Montgomery (1991) on page 291. Table 5 shows these data and its analysis with the modified Yates' algorithm and its inverse. The estimated effects stand in column "Fffect". In column (4') we have set to zero 
all nonsignificant effects and immediately get the residuals in the last column. Moreover,

- column (1) contains the estimated level-specific mean values and half main effects of $\mathrm{A}$ for the 8 combinations of $\mathrm{B}, \mathrm{C}$ and D

- column (2) contains the estimated level-specific mean values and half effects of $\mathrm{A}, \mathrm{B}$ and $\mathrm{AB}$ for the 4 combinations of $\mathrm{C}$ and D

- column (3) contains the estimated level-specific mean values and half effects of $\mathrm{A}, \mathrm{B}, \mathrm{AB}, \mathrm{C}, \mathrm{AC}, \mathrm{BC}$ and $\mathrm{ABC}$ for the 2 levels of D

All these level-specific effects are calculated in the full model, whereas we find the corresponding level-specific effects in the reduced model (without all insignificant effects) in columns (1'), (2') and ( $\left.3^{\prime}\right)$.

If we are interested in other level-specific mean values, main effects and interactions we can change the order of the rows, as is shown in Table 6 . We easily got Table 6 by sorting the first 7 columns of Table 5 with the column "B" as key. In this Table 6 the order of the factors is A, C, D and B. Hence we find for instance in column (2) the estimated level-specific mean values and half effects of A, C and $\mathrm{AC}$ for the 4 combinations of $\mathrm{D}$ and $\mathrm{B}$.

\section{Block Generation}

A complete $2^{k}$ factorial design may be run in $2^{p}$ blocks (with $p<k$ ) with a confounding pattern. To construct the blocks we can use Yates' algorithm. For that we define the complement of the pattern as the combination of all factors that are in the study but not in the pattern. For example $\mathrm{ABDF}$ is the complement of $\mathrm{CE}$ if we have $k=6$ factors $\mathrm{A}, \mathrm{B}, \mathrm{C}, \mathrm{D}, \mathrm{E}$ and $\mathrm{F}$. By setting the response of the complement of the pattern which is confounded with blocks to $2^{k-1}$ and all other responses to 0 in Yates' algorithm, we can then easily see the two blocks in the effect column: block 1 consists of all factor combinations with a positive entry ( 0.5 or 1$)$ and block 2 of all those with a negative entry $(-1)$. 


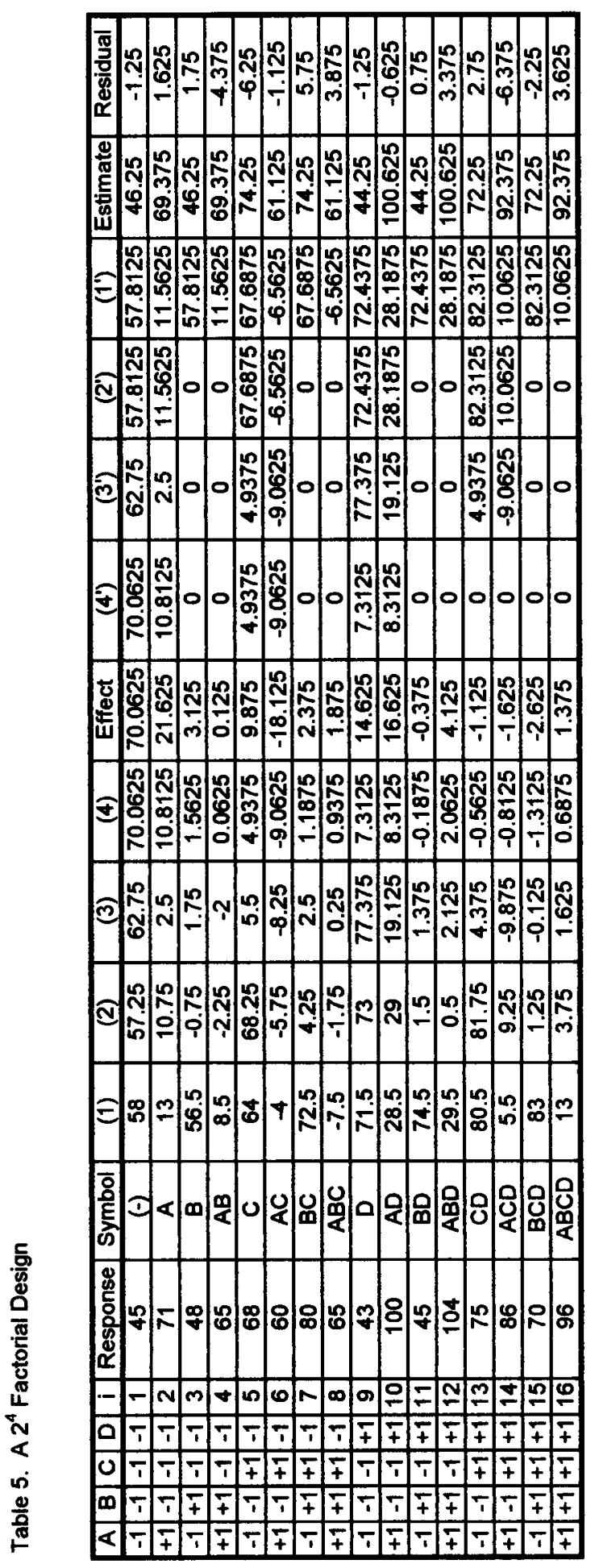




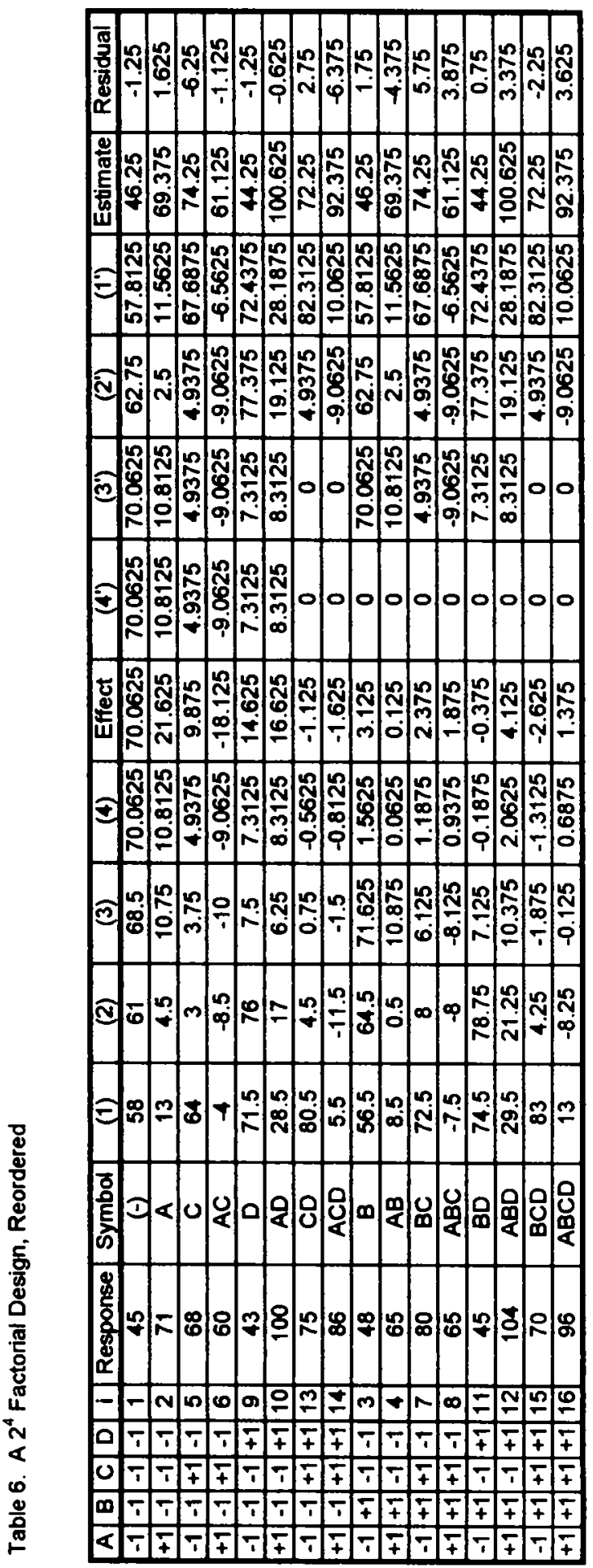


As a first example we have Table 7 with $k=3$ factors and blocks confounded with $\mathrm{ABC}$. The entry 4 in row $(-)$, which is the complement of $\mathrm{ABC}$, leads to the two blocks $(-), \mathrm{AB}, \mathrm{AC}$ and $\mathrm{BC}$ with a positive effect and $\mathrm{A}, \mathrm{B}, \mathrm{C}$ and $\mathrm{ABC}$ with a negative effect.

Table 7 Block Generator

\begin{tabular}{|c|c|c|c|c|c|}
\hline Response & Symbol & $(1)$ & $(2)$ & $(3)$ & Effect \\
\hline 4 & $(-)$ & 2 & 1 & 0.5 & 0.5 \\
\hline 0 & $\mathrm{~A}$ & -2 & -1 & -0.5 & -1 \\
\hline 0 & $\mathrm{~B}$ & 0 & -1 & -0.5 & -1 \\
\hline 0 & $\mathrm{AB}$ & 0 & 1 & 0.5 & 1 \\
\hline 0 & $\mathrm{C}$ & 0 & 0 & -0.5 & -1 \\
\hline 0 & $\mathrm{AC}$ & 0 & 0 & 0.5 & 1 \\
\hline 0 & $\mathrm{BC}$ & 0 & 0 & 0.5 & 1 \\
\hline 0 & $\mathrm{ABC}$ & 0 & 0 & -0.5 & -1 \\
\hline
\end{tabular}

Table 8. 4 Blocks in a $2^{5}$ Factorial Design

\begin{tabular}{|c|c|c|c|}
\hline ADE & BCE & Block & Symbol \\
\hline 0.5 & 0.5 & 1 & $(-)$ \\
\hline-1 & 1 & 2 & A \\
\hline 1 & -1 & 3 & B \\
\hline-1 & -1 & 4 & $\overline{A B}$ \\
\hline 1 & -1 & 3 & C \\
\hline-1 & -1 & 4 & $\overline{A C}$ \\
\hline 1 & 1 & 1 & $B C$ \\
\hline-1 & 1 & 2 & $\overline{A B C}$ \\
\hline-1 & 1 & 2 & $\mathrm{D}$ \\
\hline 1 & 1 & 1 & $A D$ \\
\hline-1 & -1 & 4 & BD \\
\hline 1 & -1 & 3 & $A B D$ \\
\hline-1 & -1 & 4 & $C D$ \\
\hline 1 & -1 & 3 & $A C D$ \\
\hline-1 & 1 & 2 & $B C D$ \\
\hline 1 & 1 & 1 & $A B C D$ \\
\hline-1 & -1 & 4 & $\bar{E}$ \\
\hline 1 & -1 & 3 & $A E$ \\
\hline-1 & 1 & 2 & $\mathrm{BE}$ \\
\hline 1 & 1 & 1 & $A B E$ \\
\hline-1 & 1 & 2 & $C E$ \\
\hline 1 & 1 & 1 & ACE \\
\hline-1 & -1 & 4 & $\mathrm{BCE}$ \\
\hline 1 & -1 & 3 & ABCE \\
\hline 1 & -1 & 3 & $\mathrm{DE}$ \\
\hline-1 & -1 & 4 & ADE \\
\hline 1 & 1 & 1 & BDE \\
\hline-1 & 1 & 2 & ABDE \\
\hline 1 & 1 & 1 & $\mathrm{CDE}$ \\
\hline-1 & 1 & 2 & ACDE \\
\hline 1 & -1 & 3 & BCDE \\
\hline-1 & -1 & 4 & $A B C D E$ \\
\hline
\end{tabular}


As a second example we construct the 4 blocks in a $2^{5}$ factorial design that confounds the effects $A D E, B C E$ and $A B C D$ with block effects. We set first the response of the complement $\mathrm{BC}$ of ADE equal to 16 and all other responses zero and get as "Effect" the column labelled "ADE" in Table 8. The following column labelled "BCE" is the "Effect" column after setting the "Response" of the complement $\mathrm{AD}$ of $\mathrm{BCE}$ equal to 16 and all other responses zero in Yates' algorithm. The sign pattern of columns "ADE" and "BCE" defines the blocks: number 1 for two positive numbers, 2 for $(-1,+1), 3$ for $(+1,-1)$ and 4 for $(-1,-1)$. The same pattern could be generated by any pair of the 3 effects which are confounded with blocks. This method of generating blocks easily generalizes to higher order factorial designs and even more blocks.

\section{Generating the Layout Sheet of a Fractional Factorial Design and Analysis of the Response}

If a $2^{k}$ design should be split in two one-half fractions, we can generate the layout sheet once again by Yates' algorithm. If for example the design generator is given by $\mathrm{I}=\mathrm{ABC}$, then we set the response equal to $2^{k-2}$ for the treatment combination (-) and $-2^{k-2}$ for the interaction $\mathrm{ABC}$. The principal fraction consists of all factor combinations that now have a entry of 0 in the "Effect" column, and to the alternate fraction belong all those with entry +1 or -1 .

As an example we have Table 9 with $k=3$ factors. In the principal fraction are the combinations $(-), A B, A C$ and $B D$, in the

Table 9 Fractional Factorial Generator

\begin{tabular}{|c|c|c|c|c|c|}
\hline Response & Symbod & $(1)$ & $(2)$ & $(3)$ & Effect \\
\hline 2 & $(-)$ & 1 & 0.5 & 0 & 0 \\
\hline 0 & $\mathrm{~A}$ & -1 & -0.5 & -0.5 & -1 \\
\hline 0 & $\mathrm{~B}$ & 0 & -0.5 & -0.5 & -1 \\
\hline 0 & $\mathrm{AB}$ & 0 & 0.5 & 0 & 0 \\
\hline 0 & $\mathrm{C}$ & 0 & -0.5 & -0.5 & -1 \\
\hline 0 & $\mathrm{AC}$ & 0 & -0.5 & 0 & 0 \\
\hline 0 & $\mathrm{BC}$ & -1 & -0.5 & 0 & 0 \\
\hline-2 & $\mathrm{ABC}$ & -1 & -0.5 & -0.5 & -1 \\
\hline
\end{tabular}


alternate fraction $\mathrm{A}, \mathrm{B}, \mathrm{C}$ and $\mathrm{ABC}$.

As next example we generate a $2^{6-2}$ design with $\mathrm{I}=\mathrm{ABCE}$ and $\mathrm{I}=\mathrm{BCDF}$ as design generators and build two blocks confounded with the $\mathrm{ABD}$ interaction. By setting the response +16 for the treatment combination (-) and -16 for $\mathrm{ABCE}$ we get the effects in column 1 of Table 10. Then we change the response -16 from ABCE to BCDF and get column 2 in Table 10 . Then we generate column 3 , the blocks with the ABD interaction, by setting all responses equal to 0 with the exception of a 32 for CEF (the complement of ABD). The treatment combinations with 0 in the first and second column define the principal fraction and the sign of column 3 splits these into 2 blocks. There are of course three alternate fractions of this design, namely those with $( \pm 1,0),(0, \pm 1)$ and $( \pm 1, \pm 1)$ in the first two columns. Each of these alternate fractions is again split in two blocks by the signs of column 3 .

To generate the confounding pattern of the fractional factorial design we can also take the advantage of Yates' algorithm: We enter random numbers (e.g. uniformly distributed between 0 and $2^{k-2}$ ) in the column "Response" and get random effects in the column "Effect". Now we can easily study the whole alias structure for this design: factor combinations with equal random effects are confounded. There is one exception to this: To find the factors confounded with $(-)$, we have to double the random effect of (-). As an example we see in Table 10 , our $2^{6-2}$ design with 2 blocks, that (-) is confounded with $\mathrm{ABCE}, \mathrm{BCDF}$ and $\mathrm{ADEF}$ (with random effects 3.31726), and $A$ is confounded with $\mathrm{BCE}, \mathrm{ABCDF}$ and $\mathrm{DEF}$ (each with random effect -0.22665$)$.

In Table 11 we analyse the data by Montgomery (1991) given on page 352 , which form a $2^{6-2}$ design with 2 blocks, the same design as in Table 10. We put the response in a full $2^{6}$ spreadsheet with zeros for all treatment combinations not in the principal fraction. Because we have only $1 / 4$ of the responses we get $1 / 4$ of the effects in column "Effect". If we now change all non significant effects to zero, we get by the inverse algorithm the estimates and the residuals in the last columns. 
Table 10. Generation of a $2^{6-2}$ Design with 2 Blocks

\begin{tabular}{|c|c|c|c|c|c|c|}
\hline FABCE & $F=B C D F$ & $A B D$ & Block & Response & Symbol & Effect \\
\hline 0 & 0 & 0.5 & 1 & 4.766747 & $(-)$ & 1.65863 \\
\hline 1 & 0 & -1 & & 0 & A & -0.22665 \\
\hline 1 & 1 & -1 & & 0 & B & 0.53287 \\
\hline 0 & -1 & 1 & & 0 & AB & 0.59607 \\
\hline 1 & 1 & 1 & & 0 & C & 0.09819 \\
\hline 0 & -1 & -1 & & 0 & $A C$ & 0.04756 \\
\hline 0 & 0 & -1 & 2 & 3.090915 & $B C$ & -0.14730 \\
\hline 1 & 0 & 1 & & 0 & ABC & -0.09801 \\
\hline 0 & 1 & -1 & & 0 & D & 0.40869 \\
\hline-1 & -1 & 1 & & 0 & $A D$ & -0.24572 \\
\hline-1 & 0 & 1 & & 0 & BD & 0.13884 \\
\hline 0 & 0 & -1 & 2 & 5.571947 & ABD & -0.00902 \\
\hline-1 & 0 & -1 & & 0 & $C D$ & 0.92412 \\
\hline 0 & 0 & 1 & 1 & 7.478744 & $A C D$ & 0.66559 \\
\hline 0 & 1 & 1 & & 0 & BCD & 0.55600 \\
\hline-1 & -1 & -1 & & 0 & $A B C D$ & -0.31979 \\
\hline 1 & 0 & 1 & & 0 & $E$ & -0.09801 \\
\hline 0 & 0 & -1 & 2 & 5.781915 & $\mathbf{A E}$ & -0.14730 \\
\hline 0 & -1 & -1 & & 0 & $\mathrm{BE}$ & 0.04756 \\
\hline 1 & 1 & 1 & & 0 & ABE & 0.09819 \\
\hline 0 & -1 & 1 & & 0 & CE & 0.59607 \\
\hline 1 & 1 & -1 & & 0 & ACE & 0.53287 \\
\hline 1 & 0 & -1 & & 0 & BCE & -0.22665 \\
\hline 0 & 0 & 1 & 1 & 4.002564 & ABCE & 3.31726 \\
\hline-1 & -1 & -1 & & 0 & DE & -0.31979 \\
\hline 0 & 1 & 1 & & 0 & ADE & 0.55600 \\
\hline 0 & 0 & 1 & 1 & 6.294626 & BDE & 0.66559 \\
\hline-1 & 0 & -1 & & 0 & ABDE & 0.92412 \\
\hline 0 & 0 & -1 & 2 & 7.192602 & CDE & -0.00902 \\
\hline-1 & 0 & 1 & & 0 & ACDE & 0.13884 \\
\hline-1 & -1 & 1 & & 0. & BCDE & -0.24572 \\
\hline 0 & 1 & -1 & & 0 & ABCDE & 0.40869 \\
\hline 0 & 1 & 1 & & 0 & $F$ & 0.55600 \\
\hline-1 & -1 & -1 & & 0 & AF & -0.31979 \\
\hline-1 & 0 & -1 & & 0 & BF & 0.92412 \\
\hline 0 & 0 & 1 & 1 & 11.70446 & ABF & 0.66559 \\
\hline-1 & 0 & 1 & & 0 & CF & 0.13884 \\
\hline 0 & 0 & -1 & 2 & 1.932188 & ACF & -0.00902 \\
\hline 0 & 1 & -1 & & 0 & BCF & 0.40869 \\
\hline-1 & -1 & 1 & & 0 & $A B C F$ & -0.24572 \\
\hline 0 & 0 & -1 & 2 & 9.262001 & DF & -0.14730 \\
\hline 1 & 0 & 1 & & 0 & $\overline{A D F}$ & -0.09801 \\
\hline 1 & 1 & 1 & & 0 & BDF & 0.09819 \\
\hline 0 & -1 & -1 & & 0 & ABDF & 0.04756 \\
\hline 1 & 1 & -1 & & 0 & CDF & 0.53287 \\
\hline 0 & -1 & 1 & & 0 & ACDF & 0.59607 \\
\hline 0 & 0 & 1 & 1 & 10.83724 & BCDF & 3.31726 \\
\hline 1 & 0 & -1 & & 0 & ABCDF & -0.22665 \\
\hline-1 & -1 & 1 & & 0 & EF & -0.24572 \\
\hline 0 & 1 & -1 & & 0 & AEF & 0.40869 \\
\hline 0 & 0 & -1 & 2 & 7.622791 & BEF & -0.00902 \\
\hline-1 & 0 & 1 & & 0 & ABEF & 0.13884 \\
\hline 0 & 0 & 1 & 1 & 7.635487 & CEF & 0.66559 \\
\hline-1 & 0 & -1 & & 0 & ACEF & 0.92412 \\
\hline-1 & -1 & -1 & & 0 & BCEF & 0.31979 \\
\hline 0 & 1 & 1 & & 0 & ABCEF & 0.55600 \\
\hline 1 & 0 & -1 & & 0 & DEF & -0.22665 \\
\hline 0 & 0 & 1 & 1 & 0.500504 & ADEF & 3.31726 \\
\hline 0 & -1 & 1 & & 0 & BOEF & 0.59607 \\
\hline 1 & 1 & -1 & & 0 & ABDEF & 0.53287 \\
\hline 0 & -1 & -1 & & 0 & CDEF & 0.04756 \\
\hline 1 & 1 & 1 & & 0 & ACDEF & 0.09819 \\
\hline 1 & 0 & 1 & & 0 & BCDEF & -0.09801 \\
\hline 0 & 0 & -1 & 2 & 12.47743 & ABCDEF & -0.14730 \\
\hline
\end{tabular}


Table 11 Analysis of a $2^{6-2}$ Design with 2 Blocks

\begin{tabular}{|c|c|c|c|c|}
\hline Response & Symbol & Effect & Estimate & Residual \\
\hline \multirow[t]{6}{*}{6} & $(-)$ & 6.828125 & 8.5 & -2.5 \\
\hline & A & 3.46875 & 0 & 0 \\
\hline & B & 8.90625 & 0 & 0 \\
\hline & $A B$ & 2.96875 & 0 & 0 \\
\hline & C & 0 & 0 & 0 \\
\hline & $A C$ & 0 & 0 & 0 \\
\hline \multirow[t]{5}{*}{26} & $B C$ & 0 & 32.25 & -6.25 \\
\hline & $A B C$ & 0 & 0 & 0 \\
\hline & D & 0 & $\underline{0}$ & 0 \\
\hline & $A D$ & 0 & 0 & 0 \\
\hline & BD & 0 & 0 & 0 \\
\hline \multirow[t]{2}{*}{60} & ABD & 0 & 58 & 2 \\
\hline & CD & 0 & 0 & 0 \\
\hline \multirow[t]{4}{*}{5} & $A C D$ & 0 & 10.5 & -5.5 \\
\hline & $B C D$ & 0 & 0 & 0 \\
\hline & $A B C D$ & 0 & 0 & 0 \\
\hline & $\mathbf{E}$ & 0 & 0 & 0 \\
\hline \multirow[t]{6}{*}{10} & $A E$ & 0 & 10.5 & -0.5 \\
\hline & BE & 0 & 0 & 0 \\
\hline & $A B E$ & 0 & 0 & 0 \\
\hline & $\mathrm{CE}$ & 2.96875 & D & 0 \\
\hline & ACE & 8.90625 & 0 & 0 \\
\hline & BCE & 3.46875 & 0 & 0 \\
\hline \multirow[t]{3}{*}{60} & ABCE & 13.65625 & 58 & 2 \\
\hline & DE & 0 & 0 & 0 \\
\hline & ADE & 0 & 0 & 0 \\
\hline \multirow[t]{2}{*}{34} & BDE & 0 & 32.25 & 1.75 \\
\hline & ABDE & 0 & D & 0 \\
\hline \multirow[t]{7}{*}{16} & CDE & 0 & 8.5 & 7.5 \\
\hline & ACDE & 0 & 0 & 0 \\
\hline & BCDE & 0 & 0 & 0 \\
\hline & $A B C D E$ & 0 & 0 & 0 \\
\hline & $F$ & 0 & 0 & 0 \\
\hline & AF & 0 & 0 & 0 \\
\hline & $B F$ & 0 & 0 & 0 \\
\hline \multirow[t]{2}{*}{60} & ABF & 0 & 58 & 2 \\
\hline & $C F$ & 0 & 0 & 0 \\
\hline \multirow[t]{3}{*}{15} & ACF & 0 & 10.5 & 4.5 \\
\hline & BCF & 0 & 0 & 0 \\
\hline & ABCF & 0 & 0 & 0 \\
\hline \multirow[t]{6}{*}{8} & DF & 0 & 8.5 & -0.5 \\
\hline & ADF & 0 & 0 & 0 \\
\hline & BDF & 0 & 0 & 0 \\
\hline & ABDF & 0 & 0 & 0 \\
\hline & CDF & 8.90625 & 0 & 0 \\
\hline & ACDF & 2.96875 & 0 & 0 \\
\hline \multirow[t]{4}{*}{37} & BCDF & 13.65625 & 32.25 & 4.75 \\
\hline & ABCDF & 3.46875 & 0 & 0 \\
\hline & EF & 0 & 0 & 0 \\
\hline & AEF & 0 & 0 & 0 \\
\hline \multirow[t]{2}{*}{32} & BEF & 0 & 32.25 & -0.25 \\
\hline & ABEF & 0 & 0 & 0 \\
\hline \multirow[t]{5}{*}{4} & CEF & $\underline{0}$ & 8.5 & -4.5 \\
\hline & ACEF & 0 & 0 & 0 \\
\hline & BCEF & 0 & 0 & 0 \\
\hline & ABCEF & 0 & 0 & 0 \\
\hline & DEF & 3.46875 & 0 & 0 \\
\hline 12 & ADEF & 13.65625 & 10.5 & 1.5 \\
\hline & BDEF & 2.95875 & 0 & 0 \\
\hline & ABDEF & 8.90625 & 0 & 0 \\
\hline & CDEF & 0 & 0 & 0 \\
\hline & ACDEF & 0 & 0 & 0 \\
\hline & BCDEF & 0 & 0 & 0 \\
\hline 52 & ABCDEF & 0 & 58 & -6 \\
\hline
\end{tabular}




\section{Appendix A: Construction of the Modified Algorithm and its Inverse}

In order to get the modified algorithm with $\mathrm{k}$ factors, we proceed as follows: We begin with one factor and add a second, third ... adapting in each step the table of calculations.

The following spreadsheet-like table of calculations shows the algorithm in its most simple form with $k=1$ factor:

Response Symbol

A1

A2

A

$\mathrm{B} 1=(\mathrm{A} 1+\mathrm{A} 2) / 2$

$\mathrm{B} 2=(\mathrm{A} 2-\mathrm{A} 1) / 2$

Starting with the table of calculations with $\mathrm{k}$ factors which has $2^{k}$ rows, we can construct the one with $k+1$ factors in the following three steps:

1. We add a new column $(k+1)$

2. We copy the whole table below itself. The table now has $2^{k+1}$ rows. In the lower half we add the symbol of a new factor in every cell of column "Symbol".

3. The cells in the column $(\mathrm{k}+1)$ are constructed as follows: in row $j$ for $j=1,2, \ldots, 2^{k} \quad$ we calculate the mean of the cells $\mathrm{j}$ and $j+2^{k}$ of column (k)

in row $2^{k}+j$ for $j=1,2, \ldots, 2^{k}$ we calculate half the difference of the cells $2^{k}+j$ and $\mathrm{j}$ of column ( $\left.\mathrm{k}\right)$

For example, starting from the table above with $k=1$ we get the following one with 2 factors:

Response Symbol (1)

A1

A2

A3

A4
$(-)$

A

B

AB
$\mathrm{B} 1=(\mathrm{A} 1+\mathrm{A} 2) / 2$

$\mathrm{B} 2=(\mathrm{A} 2-\mathrm{A} 1) / 2$

$\mathrm{B} 3=(\mathrm{A} 3+\mathrm{A} 4) / 2$

$\mathrm{B} 4=(\mathrm{A} 4-\mathrm{A} 3) / 2$
(2)

$\mathrm{C} 1=(\mathrm{B} 1+\mathrm{B} 3) / 2$

$\mathrm{C} 2=(\mathrm{B} 2+\mathrm{B} 4) / 2$

$\mathrm{C} 3=(\mathrm{B} 3-\mathrm{B} 1) / 2$

$\mathrm{C} 4=(\mathrm{B} 4-\mathrm{B} 2) / 2$ 
Note that the column "Effect" is always constructed by doubling all entries in the last column (k), with the exception of the first row which has the same eritry in columns (k) and "Effect".

The inverse algorithn is closely related to the modified Yates' algorithm: we take back every step that has been done, beginning with the last one. The step from column "Effect" to column ( $k$ ') makes just the opposite of the one from (k) to "Effect": it divides in half all the estimated main effects and interactions and leaves unchanged the estimated mean. Similarly the calculations from (k') to (k-1') take back those from (k-1) to (k): we have to drop the division by 2 , interchange all the plus and minus signs and interchange the subtrahend and the subtractor in every subtraction. In this manner we inverse one by one ali the calculation steps in the modified Yates' algorithm and finally get column "Estimate".

As an illustration, we give the table of calculations for the inverse algorithm with two factors:

\begin{tabular}{|c|c|c|c|c|}
\hline mbal & Effect & $\left(2^{\prime}\right)$ & $\left(1^{\prime}\right)$ & nate \\
\hline-$)$ & D1 & $\mathrm{E} 1=\mathrm{D} 1$ & $\mathrm{~F} 1=\mathrm{E} 1-\mathrm{E} 3$ & $\mathrm{G} 1=\mathrm{F} 1-\mathrm{F}$ \\
\hline$i$ & D2 & $\mathrm{E} 2=$ & $-\mathrm{E} 4$ & \\
\hline & D3 & $\mathrm{E} 3=$ & $3+\mathrm{E} 1$ & G3 \\
\hline D & D4 & $\mathrm{E} 4=\mathrm{D} 4 / 2$ & $\mathrm{~F} 4=\mathrm{E} 4+\mathrm{E} 2$ & G4 \\
\hline
\end{tabular}

\section{Appendix B}

In this Appendix we will prove that our methods of constructing the blocks and the layout sheets in fractional factorial designs are equivalent to the standard methods.

\subsection{The Function $f_{k}$}

Let $\mathrm{k}$ be the number of factors $\mathrm{A}, \mathrm{B}, \mathrm{C}, \ldots$ in the study. Every factor combination can be represented by a vector $x=\left(x_{1}, x_{2}, \ldots, x_{k}\right)$ with $x_{j} \in\{0,1\}$, where $x_{:}=1$ and 0 stand for the level +1 or -1 of the $\mathrm{j}$-th factor respectiveiy. For example $(1,0,1)$ represents $\mathrm{AC}$ in a study with 3 factors.

Definition: The function $f_{k}$ is defined as 


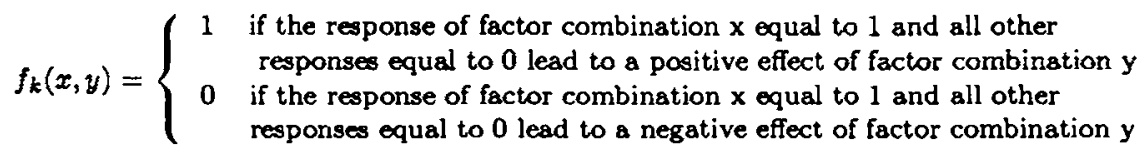

This definition is clarified by Table B.1, where we obviously have $q \neq 0$, and further

$$
q>0 \Leftrightarrow f_{k}(x, y)=1 \text { and } q<0 \Leftrightarrow f_{k}(x, y)=0 .
$$

Table B.1: Clarifying the function $f_{k}$

\begin{tabular}{|c|c|c|c|c|c|}
\hline Factor Comb. & Response & $(1)$ & $\ldots$ & $(\mathrm{k}-1)$ & $(\mathrm{k})$ \\
\hline$\vdots$ & 0 & & & & \\
$\vdots$ & $\vdots$ & & & & \\
\hline$\left(x_{1}, x_{2}, \ldots, x_{k}\right)$ & 1 & & & & \\
\hline$\cdot$ & 0 & & & & \\
\hline$\left(y_{1}, y_{2}, \ldots, y_{k}\right)$ & 0 & & & & \\
\hline$\vdots$ & $\vdots$ & & & & \\
\hline
\end{tabular}

Table B.2

\begin{tabular}{|c|c|c|}
\hline Factor Comb. & Response & Effect \\
\hline$(-)$ & 1 & +0.5 \\
$\mathrm{~A}$ & 0 & -1 \\
\hline
\end{tabular}

Table B.3

\begin{tabular}{|c|c|c|}
\hline Factor Comb. & Response & Effect \\
\hline$(-)$ & 0 & +0.5 \\
$\mathrm{~A}$ & 1 & +1 \\
\hline
\end{tabular}

Theorem: $f_{k}(x, y)=1+\sum_{j=1}^{k} y_{j}+\sum_{j=1}^{k} x_{j} y_{j} \quad \bmod 2$

Proof: This theorem is proved by induction. For $k=1$ we can see in Tables B.2 and B.3 that the function $f_{1}\left(x_{1}, y_{1}\right)$ can be displayed as

\begin{tabular}{|ll||l|l|}
\hline & $x_{1}$ & 0 & 1 \\
$y_{1}$ & & & \\
\hline \hline 0 & & 1 & 1 \\
\hline 1 & & 0 & 1 \\
\hline
\end{tabular}


and can therefore be written as $f_{1}\left(x_{1}, y_{1}\right)=1+y_{1}+x_{1} y_{1} \bmod 2$

Table B.4 will help us with the step from $k$ to $k+1$ factors. Note that colums $(k+1)$ and "Effect" are the same up to a factor 2 and hence have the same sign.

Table B.4: The step from $k$ to $k+1$

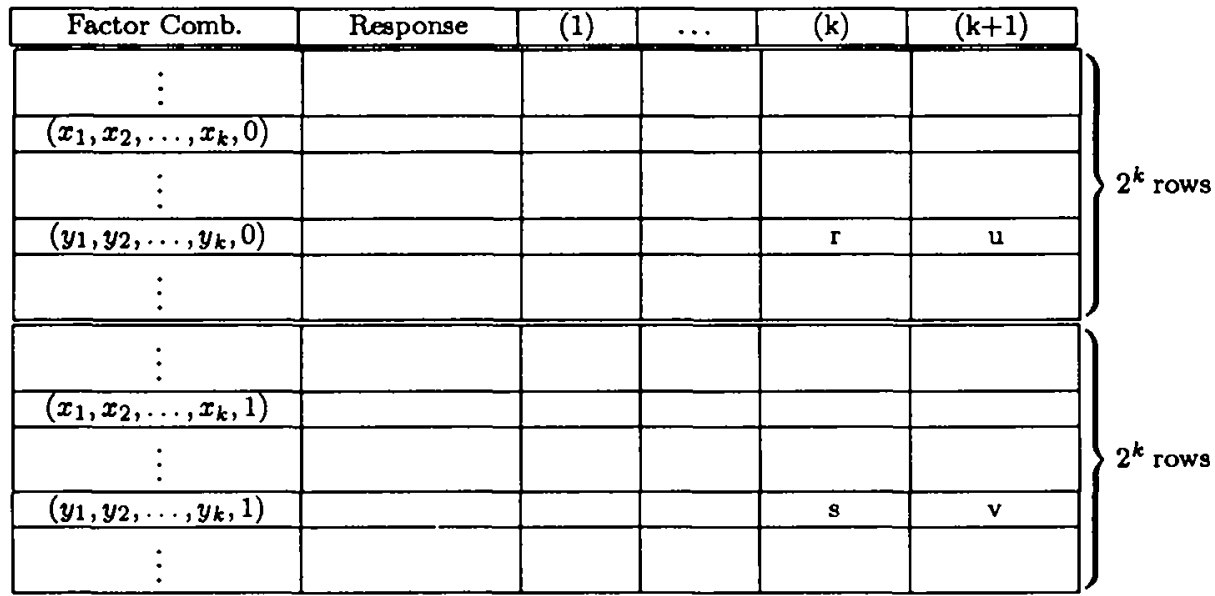

Analysing the way the tables of calculations are constructed (see Appendix A), we find $u=(r+s) / 2$ and $v=(s-r) / 2$.

We now look at two different cases:

Case 1: $x_{k+1}=0$

In this case, the only response which is unequal to 0 is $\left(x_{1}, \ldots, x_{k}, 0\right)$ which is in the upper half. Hence $r \neq 0, s=0, u=r / 2$ and $v=-r / 2$.

Therefore we have $f_{k+1}\left(x_{1}, \ldots, x_{k}, 0, y_{1}, \ldots, y_{k}, 0\right)=1$

$$
\begin{aligned}
& \Leftrightarrow u>0 \Leftrightarrow r>0 \\
& \Leftrightarrow f_{k}\left(x_{1}, \ldots, x_{k}, y_{1}, \ldots, y_{k}\right)=1
\end{aligned}
$$

and $f_{k+1}\left(x_{1}, \ldots, x_{k}, 0, y_{1}, \ldots, y_{k}, 1\right)=1$

$$
\Leftrightarrow v>0 \Leftrightarrow r<0 \Leftrightarrow f_{k}\left(x_{1}, \ldots, x_{k}, y_{1}, \ldots, y_{k}\right)=0
$$

Case 2: $x_{k+1}=1$

Similarly, we find $r=0, s \neq 0, u=s / 2$ and $v=s / 2$. 


$$
\begin{aligned}
& f_{k+1}\left(x_{1}, \ldots, x_{k}, 1, y_{1}, \ldots, y_{k}, 0\right)=1 \\
& \Leftrightarrow u>0 \Leftrightarrow s>0 \Leftrightarrow f_{k}\left(x_{1}, \ldots, x_{k}, y_{1}, \ldots, y_{k}\right)=1 \\
& \text { and } f_{k+1}\left(x_{1}, \ldots, x_{k}, 1, y_{1}, \ldots, y_{k}, 1\right)=1 \\
& \quad \Leftrightarrow v>0 \Leftrightarrow s>0 \Leftrightarrow f_{k}\left(x_{1}, \ldots, x_{k}, y_{1}, \ldots, y_{k}\right)=1
\end{aligned}
$$

Summing up we find

$$
\begin{aligned}
& f_{k+1}\left(x_{1}, \ldots, x_{k}, x_{k+1}, y_{1}, \ldots, y_{k}, y_{k+1}\right) \\
& \quad \equiv f_{k}\left(x_{1}, \ldots, x_{k}, y_{1}, \ldots, y_{k}\right)+\left(1+x_{k+1}\right) y_{k+1} \bmod 2 \\
& \equiv 1+\sum_{j=1}^{k} y_{j}+\sum_{j=1}^{k} x_{j} y_{j}+y_{k+1}+x_{k+1} y_{k+1} \bmod 2 \\
& \equiv 1+\sum_{j=1}^{k+1} y_{j}+\sum_{j=1}^{k+1} x_{j} y_{j} \bmod 2
\end{aligned}
$$

which completes the proof.

\subsection{Constructing the Blocks}

The standard method for constructing a block design with $\mathrm{k}$ factors where the factor combination $\mathrm{x}$ is confounded with blocks consists of finding all factor combinations $y$ for which

$$
x \cdot y=\sum_{j=1}^{k} x_{j} y_{j} \equiv 0 \quad \bmod 2
$$

for block 1 and

$$
x \cdot y=\sum_{j=1}^{k} x_{j} y_{j} \equiv 1 \quad \bmod 2
$$

for block 2. See for example Montgomery (1991, p. 321).

Our method places all factor combinations $y$ in block 1 and 2 for which there is a positive and negative effect respectively if the response of factor combination $1-x$ (the complement of $\mathrm{x}$ ) is equal to 1 and all other responses are equal to 0 . Hence we place in block 1 all $y$ for which 


$$
\begin{gathered}
f_{k}(1-x, y) \equiv 1 \bmod 2 \\
\Leftrightarrow 1+\sum_{j=1}^{k} y_{j}+\sum_{j=1}^{k}\left(1-x_{j}\right) y_{j} \equiv 1 \bmod 2 \\
\Leftrightarrow \sum_{j=1}^{k} x_{j} y_{j} \equiv 0 \bmod 2
\end{gathered}
$$

Therefore our method is equivalent to the standard method.

\subsection{Constructing the Layout Sheets}

The standard method for finding the layout sheet in a fractional factorial design with $\mathrm{k}$ factors with identity generator $(0, \ldots, 0)=x$ (for example $(0,0,0)=(1,0,1)$ which is normally written as $I=$ $A C$ ) consists of finding all factor combinations y for which

$$
x \cdot y=\sum_{j=1}^{k} x_{j} y_{j} \equiv 0 \quad \bmod 2 .
$$

See for example Lorenzen and Anderson (1993, p. 225).

Our method places all factor combinations y in the principal fraction, which have an effect of 0 when the responses of $(0, \ldots, 0)$ and $\mathrm{x}$ are equal to $+2^{k-2}$ and $-2^{k-2}$ respectively and all other responses are equal to 0 .

Hence we choose all y for which

$$
\begin{gathered}
f_{k}(0, y)=f_{k}(x, y) \\
\Leftrightarrow f_{k}(0, y)+f_{k}(x, y) \equiv 0 \bmod 2 \\
\Leftrightarrow 1+\sum_{j=1}^{k} y_{j}+1+\sum_{j=1}^{k} y_{j}+\sum_{j=1}^{k} x_{j} y_{j} \equiv 0 \bmod 2 . \\
\Leftrightarrow \sum_{j=1}^{k} x_{j} y_{j} \equiv 0 \bmod 2 .
\end{gathered}
$$

Therefore our method is equivalent to the standard method. 
Acknowledgement: The author thanks Stephen Vardeman for helpful comments and Roger Hämmerli for editorial help.

\section{References}

Box, G.E.P., Hunter, W.G. and Hunter, J.S. (1978), Statistics for Experimenters, New York: John Wiley.

Daniel, C. (1976), Applications of Statistics to Industrial Experimentation, New York: John Wiley.

Lorenzen, T.J. and Anderson, V.L. (1993), Design of Experiments - A No-Name Approach, New York: Dekker.

Montgomery, D.C. (1991), Design and Analysis of Experiments, third edition, New York: John Wiley.

National Bureau of Standards (1957), Fractional Factorial Experimental Designs for Factors at Two Levels (National Bureau of Standards Applied Mathematics Series, Vol. 48), Washington DC: U.S. Government Printing Office.

Yates, F. (1935), "Complex Experiments", Journal of the Royal Statistical Society, Ser. B, 2, 181-223

Hans Riedwyl

Institute of Mathematical Statistics and Actuarial Science

University of Berne

Sidlerstraße 5

CH-3012 Bern, Switzerland 